\section{Adaptive Variable Structure Tracking Control for Constrained Robots}

CHUN-YI SU, Member, IEEE

YURY STEPANENKO,

University of Victoria

The problem of controller design for constrained robots with the consideration of computational efficiency is addressed. An efficient adaptive variable structure control algorithm, based on a reduced dynamics formulation, is presented for trajectory tracking of an end-effector on a constrained surface with specified constraint forces. It is shown that the objective can be achieved without exact knowledge of robot dynamics and on-line calculation of nonlinear dynamic functions. The control algorithm is constructed with at most three control parameters to be adjusted adaptively, and that number is determined independently of the number of degrees of freedom of the robotic manipulators. $A$ numerical example is presented to illustrate the developed method.

Manuscript received September 1, 1992; revised February 28, 1993. IEEE Log No. T-AES/30/2/15497.

Authors' address: Department of Mechanical Engineering, University of Victoria, Victoria B.C., V8W 3P6, Canada.

$\overline{0018-9251 / 94 / \$ 4.00 ~(C) ~} 1994$ IEEE

\section{INTRODUCTION}

In order to achieve a wider class of tasks which involve contact with a manipulator environment, issues of appropriate modeling and of effective new control strategies arise since such a contact usually results in the generation of external forces acting on the end-effector of a manipulator and modifies the dynamic behavior of the manipulator. Mathematical model of a robot system in contact.with its environment, when it is described by a holonomic smooth manifold, gives rise to a mathematical system composed of differential and algebraic equations $[3,7]$. The control of such systems is called constrained robot control (see, e.g. $[8,9]$ ). The objective of control is to determine the input torques to achieve tracking for a desired trajectory on a constrained surface with specified constraint forces.

The initial study on controlling a constrained robot was in [3], where the control law was derived by expressing the constraint forces as a explicit function of the state and the input, then linearizing the system and stabilizing the linearized system by state feedback. Later the problem was addressed by using the theory of descriptor variable systems [9]. However, these methods are valid only in a neighborhood about the point of linearization. Recently, a general theoretical framework of constrained motion control was rigorously developed in [8], where a mathematical model was developed for constrained robots that explicitly incorporate the constraint description. This model was then used to develop a modified computed torque controller that gives global asymptotic stability for the position and force tracking error. However, this modified computed torque controller required exact knowledge of robot dynamics. To deal with uncertainties in the constrained robot model, adaptive controls $[1,6]$ and variable structure control [4] were developed respectively, based on the model given by [8]. Other works concerning constrained robots include [17-20]. But in these cases either the accurate models are required or the control of constraint forces is not included.

In $[13,14]$, an alternative mathematical model was developed for the constrained robots which embedded the constraint equation into the dynamic equation, resulting in an affine nonlinear system without constraints. Then the adaptive control [13] and variable structure control [14] were proposed, respectively. However, the control laws require on-line calculation of nonlinear functions of the dynamical model, hence, the computational complexity for their implementations is considerably high.

Based on the model of constrained robots established in $[13,14]$, a computationally efficient adaptive variable structure control strategy is proposed 
here to achieve trajectory tracking of the end-effector on the constrained surface with specified constraint forces for rigid robot manipulators. Compared with other adaptive methods $[1,6,13]$, the proposed controller does not require on-line calculation of robot dynamics (regressor matrix). Furthermore, the control algorithm is constructed with, at most, three control parameters to be adjusted adaptively, and that number is determined independently of the number of degrees of freedom of the robotic manipulators. Compared also with robust design methods $[4,19]$, the novelty of our result lies in the fact that uncertainty bounds, needed to derive the control law and to prove the stable tracking, are generated by simple adaptation laws. Previous results $[4,19]$ explicitly rely on uncertainty bounds that depend not only on the unknown robot parameters but also on the reference trajectory and on the robot state vector. As a result, precise bounds on the uncertainty have been difficult to compute. Therefore, the so-designed controller turns out to be very general and structurally simple as well as computationally fast, and easily extendable to a higher number of links. The robustness analysis has shown that in the presence of a class of dynamic (state-dependent) uncertainties, the control law still provides stable tracking.

This work is organized as follows: In Section II, the reduced dynamical model and its properties are briefly overviewed. Section III presents the control algorithm, and its robustness analysis is given in Section IV. Section $\mathrm{V}$ provides illustrative examples using the proposed approach. In Section VI, some conclusions are presented.

\section{CONSTRAINED ROBOT DYNAMICS}

Based on Euler-Lagrangian formulation, the motion equation of an $n$-link rigid constrained robot can be expressed in joint space as

$$
D(\mathbf{q}) \ddot{\mathbf{q}}+B(\mathbf{q}, \dot{\mathbf{q}}) \dot{\mathbf{q}}+G(\mathbf{q})=\mathbf{u}+\mathbf{f}
$$

where $\mathbf{q} \in R^{n}$ is the generalized coordinates (joint positions); $\mathbf{u} \in R^{n}$ is the vector of applied joint torques; f $\in R^{n}$ is the vector of constraint forces in joint space; $D(\mathbf{q}) \in R^{n \times n}$ is the symmetric, bounded, positive definite inertia matrix; vector $B(\mathbf{q}, \dot{\mathbf{q}}) \dot{\mathbf{q}} \in R^{n}$ presents the centripetal and Coriolis torques; $G(q) \in R^{n}$ is the vector of gravitational torques, which is bounded $C^{1}$ function.

Two simplifying properties should be noted about this dynamic structure.

Property 1. A suitable definition of $B(\mathbf{q}, \dot{\mathbf{q}})$ makes the matrix $(\dot{D}-2 B)$ skew-symmetric [10], i.e.,

$$
\mathbf{x}^{T}(\dot{D}-2 B) \mathbf{x}=0, \quad \forall \mathbf{x} \in R^{n} .
$$

In particular, this is true if the elements of $B(\mathbf{q}, \dot{\mathbf{q}})$ are defined as

$$
B_{i j}=\frac{1}{2}\left[\dot{\mathbf{q}}^{T} \frac{\partial D_{i j}}{\partial \mathbf{q}}+\sum_{k=1}^{n}\left(\frac{\partial D_{i k}}{\partial q_{j}}-\frac{\partial D_{j k}}{\partial q_{i}}\right) \dot{q}_{k}\right] .
$$

Property 2. Since $D(\mathbf{q}), G(\mathbf{q}), B(\mathbf{q}, \dot{\mathbf{q}})$ are bounded in $\mathbf{q}$ and $B(\mathbf{q}, \dot{\mathbf{q}})$ is linear in $\dot{\mathbf{q}}$, there exist positive constants $\tilde{\rho}_{i}, i=1,2,3$, such that [15]

$$
\begin{aligned}
\|D(\mathbf{q})\| & \leq \tilde{\rho}_{1} \\
\|B(\mathbf{q}, \dot{\mathbf{q}})\| & \leq \tilde{\rho}_{2}\|\dot{\mathbf{q}}\| \\
\|G(\mathbf{q})\| & \leq \tilde{\rho}_{3} .
\end{aligned}
$$

Let $p \in R^{n}$ denote the generalized position vector of the end-effector, in terms of a fixed workspace coordinate system. If the constraints imposed are described by a holonomic smooth manifold, then the algebraic equation for the constraints can be written as

$$
\phi(p)=0
$$

where the mapping $\phi: R^{n} \rightarrow R^{m}$ is twice continuously differentiable.

Assuming that the vector $p$ can be expressed in joint space by the relation

$$
p=H(\mathbf{q})
$$

where the mapping $H: R^{n} \rightarrow R^{n}$ is twice continuously differentiable. Then, the constrained equation in joint space can be written as

$$
\psi(\mathbf{q})=\phi(H(\mathbf{q}))=0 .
$$

The Jacobian matrix of the constrained equation (6) is

$$
J(\mathbf{q})=\frac{\partial \psi}{\partial \mathbf{q}}=\frac{\partial \phi}{\partial p} \frac{\partial H(\mathbf{q})}{\partial \mathbf{q}} .
$$

Since $\psi(\mathbf{q})=0$ is identically satisfied, it is evident that $J \dot{q}=0$. Thus, the effect of the constraints on the end-effector can be viewed as restricting the robot dynamics to the manifold $\Omega$ defined by

$$
\Omega=\{(\mathbf{q}, \dot{\mathbf{q}}): \psi(\mathbf{q})=0 ; J(\mathbf{q}) \dot{\mathbf{q}}=0\}
$$

rather than the space $R^{2 n}$.

When the end-effector is moving along the constrained surface, the constraint force in joint space is then given by

$$
\mathbf{f}=J^{T}(\mathbf{q}) \lambda
$$

where $\lambda \in R^{m}$ is the contact forces $[7,8]$.

Since the presence of $m$ constraints causes the manipulator to lose $m$ degrees of freedom, the manipulator is left with only $n-m$ degrees of freedom. In this case, $n-m$ linear independent coordinates are sufficient to characterize the constrained motion. Following [13, 14], choosing $n-m$ out of $n$ joint variables, denoted by

$$
\mathbf{q}^{1}=\left[q_{1}^{1} \cdots q_{n-m}^{1}\right]^{T}
$$


to be the generalized coordinates describes the constrained motion of the manipulator. The remaining joint variables are denoted by

$$
\mathbf{q}^{2}=\left[q_{1}^{2} \cdots q_{m}^{2}\right]^{T} .
$$

By the implicit function theorem, the constraint equation (6) can always be expressed explicitly as [8]

$$
\mathbf{q}^{2}=\sigma\left(\mathbf{q}^{1}\right) \text {. }
$$

It is assumed that the elements of $\mathbf{q}^{1}$ are chosen to be the first $n-m$ components of $q$. If this is not the case, (1) can always be reordered so that the first $n-m$ equations correspond to $\mathbf{q}^{1}$ and the last $m$ equations to $\mathbf{q}^{2}$.

Still following $[13,14]$, by defining

$$
L\left(\mathbf{q}^{1}\right)=\left[\begin{array}{c}
I_{n-m} \\
\frac{\partial \sigma\left(\mathbf{q}^{1}\right)}{\partial \mathbf{q}^{1}}
\end{array}\right]
$$

then, the dynamic model (1) of robots, when restricting to the constraint surface, can be expressed in a reduced form as $[13,14]$

$$
D\left(\mathbf{q}^{1}\right) L\left(\mathbf{q}^{1}\right) \ddot{\mathbf{q}}^{1}+B_{1}\left(\mathbf{q}^{1}, \dot{\mathbf{q}}^{1}\right) \dot{\mathbf{q}}^{1}+G\left(\mathbf{q}^{1}\right)=\mathbf{u}+\mathbf{f}
$$

where $B_{1}$ is defined as

$$
B_{1}\left(\mathbf{q}^{1}, \dot{\mathbf{q}}^{1}\right)=D\left(\mathbf{q}^{1}\right) \dot{L}\left(\mathbf{q}^{1}\right)+B\left(\mathbf{q}^{1}, \dot{\mathbf{q}}^{1}\right) L\left(\mathbf{q}^{1}\right) .
$$

Two fundamental properties of the dynamic equation (11) have been established in $[13,14]$ and are presented as follows.

Property 3. Define the matrix $A\left(\mathbf{q}^{1}\right)=$ $L^{T}\left(\mathbf{q}^{1}\right) D\left(\mathbf{q}^{1}\right) L\left(\mathbf{q}^{1}\right)$, then $\dot{A}\left(\mathbf{q}^{1}\right)-2 L^{T}\left(\mathbf{q}^{1}\right) B_{1}\left(\mathbf{q}^{1}, \dot{\mathbf{q}}^{1}\right)$ is skew-symmetric.

Property 4.

$$
J\left(\mathbf{q}^{1}\right) L\left(\mathbf{q}^{1}\right)=L^{T}\left(\mathbf{q}^{1}\right) J^{T}\left(\mathbf{q}^{1}\right)=0 .
$$

The above properties are fundamental for designing the force/motion control law.

\section{ADAPTIVE VARIABLE STRUCTURE CONTROLLER DESIGN}

\section{A. Controller Structure}

The considered adaptive variable structure controller (AVSC) design problem is stated as follows: Given a desired joint trajectory $\mathbf{q}_{d}$ and desired constraint force $\mathbf{f}_{d}$, or identically desired multiplier $\lambda_{d}$, satisfying the imposed constraints, i.e., $\psi\left(\mathbf{q}_{d}\right)=0$ and $\mathbf{f}_{d}=J^{T}\left(\mathbf{q}_{d}\right) \lambda_{d}$, determine a sliding mode control law such that for all $(\mathbf{q}(0), \dot{\mathbf{q}}(0)) \in \Omega, \mathbf{q} \rightarrow \mathbf{q}_{d}$, and $\mathbf{f} \rightarrow f_{d}$ as $t \rightarrow \infty$.

It should be noted that, since $\mathbf{q}^{2}=\sigma\left(\mathbf{q}^{1}\right)$, it is only required to find a control law to satisfy $\mathbf{q}^{1} \rightarrow q_{d}^{1}$ as $t \rightarrow \infty$.

In the subsequent discussion we need the following notation and definition. Specifically, the norm of vector $\mathbf{x}$ is defined as $\|\mathbf{x}\|=\left(\sum_{i=1}^{n} x_{i}^{2}\right)^{1 / 2}$ and that of matrix $A$ is defined as the corresponding induced norm $\|A\|=$ ( max $\left._{\text {eigenvalue }} A^{T} A\right)^{1 / 2}$. The singular value of matrix $A$ is defined as $\gamma(A)=\left(\text { eigenvalue }\left(A^{T} A\right)\right)^{1 / 2} \cdot \gamma_{\min }(A)$ denotes the smallest singular value. The relation $\mathbf{x}^{T} A \mathbf{x} \geq \gamma_{\min }(A)\|\mathbf{x}\|^{2}$, for $A=A^{T}>0$ concerning $\gamma(A)$ is useful in deriving the control algorithm.

To derive the AVSC, the following assumptions are required.

Assumption A1. The desired trajectory $\mathbf{q}_{d}(t)$ is chosen such that $\mathbf{q}_{d}, \dot{\mathbf{q}}_{d}$, and $\ddot{\mathbf{q}}_{d}$ are all bounded signals.

Assumption A2. The matrix $L\left(\mathbf{q}^{1}\right)$ satisfies

$$
\left\|L^{T} L\right\| \geq \beta_{1}, \quad\left\|L^{T} L\right\| \leq \beta_{2}
$$

where $\beta_{1}$ and $\beta_{2}$ are positive constants.

REMARK The assumption $\left\|L^{T} L\right\| \geq \beta_{1}$ can always be held due to the structure property of $L$.

Assumption A3. There exists a positive constant $\beta_{3}$ such that $\left\|\partial \delta\left(\mathbf{q}^{1}\right) / \partial \mathbf{q}^{1}\right\| \leq \beta_{3}$. Then, for all $\left(\mathbf{q}^{1}, \dot{\mathbf{q}}^{1}\right) \in$ $R^{2(n-m)}$, the matrices $D\left(\mathbf{q}^{1}\right), B_{1}\left(\mathbf{q}^{1}, \dot{\mathbf{q}}^{1}\right)$, and $G\left(\mathbf{q}^{1}\right)$ satisfy

$$
\begin{aligned}
\left\|L^{T} D\left(\mathbf{q}^{1}\right) L\right\| & \leq \rho_{1} \beta_{2} \\
\left\|L^{T} B_{1}\left(\mathbf{q}^{1}, \dot{\mathbf{q}}^{1}\right)\right\| & \leq \rho_{2} \beta_{2}\left\|\dot{\mathbf{q}}^{1}\right\| \\
\left\|L^{T} G\left(\mathbf{q}^{1}\right)\right\| & \leq \rho_{3} \beta_{2}
\end{aligned}
$$

where $\rho_{i}, i=1,2,3$, are positive constants.

REMARK Due to Property 1 of the robotic dynamics, and the definition of $B_{1}\left(\mathbf{q}^{1}, \dot{\mathbf{q}}^{1}\right)$ (where $\dot{L}=$ $\left(\partial \delta\left(\mathbf{q}^{1}\right) / \partial \mathbf{q}^{1}\right) \dot{\mathbf{q}}^{1}$ is also linear in $\left.\dot{\mathbf{q}}^{1}\right)$, the inequalities are valid.

A sliding manifold $s_{1}=0 \in R^{n}$ is defined as a hyperplane

$$
\mathbf{s}_{1}=\dot{\mathbf{e}}_{m}+\Lambda \mathbf{e}_{m}
$$

where $\mathbf{e}_{m}=\mathbf{q}^{1}-\mathbf{q}_{d}^{1}$ denotes the tracking error, and $\Lambda$ is a positive definite matrix whose eigenvalues are strictly in the right-half complex plane.

The AVSC is then defined as

$$
\mathbf{u}=\left\{\begin{array}{lll}
-K_{d} L \mathbf{s}_{1}-\frac{\beta_{2}}{\beta_{1}} L\left(\hat{\rho}_{1}\left\|\ddot{\mathbf{q}}_{r}^{1}\right\|+\hat{\rho}_{2}\left\|\dot{\mathbf{q}}^{1}\right\|\left\|\left.\right|_{\mathbf{q}} ^{1}\right\|+\hat{\rho}_{3}\right) \frac{\mathbf{s}_{1}}{\left\|\mathbf{s}_{1}\right\|}-J^{T}\left(\mathbf{q}^{1}\right) \lambda_{c} & \text { if } & \left\|\mathbf{s}_{1}\right\| \neq 0 \\
-J^{T}\left(\mathbf{q}^{1}\right) \lambda_{c} & \text { if } & \left\|\mathbf{s}_{1}\right\|=0
\end{array}\right.
$$


where $K_{d} \in R^{n \times n}$ is a positive definite matrix; $\hat{\rho}_{i}$, $i=1,2,3$, are the adaptive control gains; $\dot{\mathbf{q}}_{r}^{1} \in R^{n-m}$ is a vector of auxiliary signals defined by

$$
\dot{\mathbf{q}}_{r}^{1}=\dot{\mathbf{q}}_{d}^{1}-\Lambda_{d} \mathbf{e}_{m}
$$

the force term $\lambda_{c}$ in (16) is defined as

$$
\lambda_{c}=\lambda_{d}-K_{\lambda} \mathbf{e}_{\lambda}
$$

where $K_{\lambda} \in R^{m \times m}$ is a constant matrix of force control feedback gains, $\mathbf{e}_{\lambda}=\lambda-\lambda_{d}$.

The control gains $\hat{\rho}_{i}, i=1,2,3$, are adjusted by the following adaptation law

$$
\begin{aligned}
& \dot{\hat{\rho}}_{1}=\eta_{1}\left\|\mathbf{s}_{1}\right\|\left\|\ddot{\mathbf{q}}_{r}^{1}\right\| \\
& \dot{\hat{\rho}}_{2}=\eta_{2}\left\|\mathbf{s}_{1}\right\|\left\|\dot{\mathbf{q}}^{1}\right\|\left\|\dot{\mathbf{q}}_{r}^{1}\right\| \\
& \dot{\hat{\rho}}_{3}=\eta_{3}\left\|\mathbf{s}_{1}\right\|
\end{aligned}
$$

where $\eta_{i}>0, i=1,2,3$, are arbitrary constants which determine the rate of adaptation.

The control law (16) consists of three terms. The first term actually contains two terms representing potential diference (PD) control. In the second term the adaptive control gains $\hat{\rho}_{i}, i=1,2,3$, are introduced to compensate the nonlinear dynamic terms $D L, B_{1}$, and $G$. The last term represents the force control. The above AVSC only requires position, velocity, and force feedbacks.

Discussions. The controllers given in $[1,6,13]$ actually belong to the linear parameterization approach, where on-line calculation of the modeled part of the plant dynamics (regressor matrix) is required. It is a very calculation-intensive process for a plant with many degrees of freedom [12]. On the other hand, the proposed control law (16) avoids this difficulty. Furthermore, the control algorithm (16), with the help of the robot properties, is constructed with at most three control parameters to be adjusted adaptively, and that number is determined independently of the number of degrees of freedom of the robotic manipulators.

Compared with the robust methods $[4,19]$, the novelty of our result lies in the fact that uncertainty bounds, needed to derive the control law and to prove the stable tracking, are generated by simple adaptation laws. Previous results $[4,19]$ explicitly rely on uncertainty bounds that depend not only on the unknown robot parameters but also on the reference trajectory and on the robot state vector. As a result, precise bounds on the uncertainty have been difficult to compute. An improved scheme [14], however, still needs the upper bounds of the unknown robot parameters. Other works $[17,18,20]$ on the constrained motion control use quite different control laws, and require exact knowledge of robot dynamics.

It should be noticed that the control laws, given in (16) and (19)-(21), is somewhat similar to that in
[5]. Differences exist, however, because no explicit reference model is needed, and the control of the constraint forces are included.

\section{B. Stability Analysis}

Based on the sliding surface (15), using (11), and after some calculations, then the following is obtained:

$$
\begin{aligned}
D L \dot{\mathbf{s}}_{1} & =D L\left(\ddot{\mathbf{q}}^{1}-\ddot{\mathbf{q}}_{r}^{1}\right) \\
& =\mathbf{u}-D L \ddot{\mathbf{q}}_{r}^{1}-B_{1} \dot{\mathbf{q}}_{r}^{1}-G-B_{1} \mathbf{s}_{1}+J^{T} \lambda .
\end{aligned}
$$

According to Property 4 , the above equation becomes

$$
\begin{aligned}
A \dot{\mathbf{s}}_{1}= & L^{T} D L \dot{\mathbf{s}}_{1}=L^{T} \mathbf{u}-A \ddot{\mathbf{q}}_{r}^{1}-L^{T} B_{1} \dot{\mathbf{q}}_{r}^{1} \\
& -L^{T} G-L^{T} B_{1} \mathbf{s}_{1} .
\end{aligned}
$$

We now present the following stability theorem.

THEOREM 1 Consider the control law (16) with the update laws (19)-(21) in closed loop with the constrained manipulator modeled in the reduced form (11). Then, the following holds:

1) $\mathbf{q} \rightarrow \mathbf{q}_{d}$ as $t \rightarrow \infty$.

2) Steady-state force $\left(\mathbf{f}-\mathbf{f}_{d}\right)$ is bounded and inversely proportional to the norm of matrix $\left(K_{\lambda}+I\right)$.

PROOF. Let us consider the nonnegative function

$$
V(t)=\frac{1}{2} \mathbf{s}_{1}^{T} A \mathbf{s}_{1}+\frac{1}{2}\left(\sum_{i=1}^{3}\left(\rho_{i}-\hat{\rho}_{i}\right)^{2} / \eta_{i}\right) \beta_{2}
$$

where $\rho_{i}>0, i=1,2,3$, are defined in (14).

Differentiating (23) with respect to time along the solution of (22) and using Properties 3 and 4 give

$$
\begin{aligned}
\dot{V}= & \mathbf{s}_{1}^{T} A \dot{\mathbf{s}}_{1}+\frac{1}{2} \mathbf{s}_{1}^{T} \dot{A} \mathbf{s}_{1}+\beta_{2} \sum_{i=1}^{3}\left(\rho_{i}-\hat{\rho}_{i}\right)\left(-\dot{\rho}_{i}\right) / \eta_{i} \\
= & \mathbf{s}_{1}^{T}\left(L^{T} \mathbf{u}-A \ddot{\mathbf{q}}_{r}^{1}-L^{T} B_{1} \dot{\mathbf{q}}_{r}^{1}-L^{T} G-L^{T} B_{1} \mathbf{s}_{1}\right) \\
& +\frac{1}{2} \mathbf{s}_{1}^{T}\left(\frac{d}{d t} A\right) \mathbf{s}_{1}+\beta_{2} \sum_{i=1}^{3}\left(\rho_{i}-\hat{\rho}_{i}\right)\left(-\dot{\hat{\rho}}_{i}\right) / \eta_{i} \\
= & -\mathbf{s}_{1}^{T} L^{T} K_{d} L \mathbf{s}_{1}-\frac{\beta_{2}}{\beta_{1}} \mathbf{s}_{1}^{T} L^{T} L\left(\hat{\rho}_{1}\left\|\ddot{\mathbf{q}}_{r}^{1}\right\|+\hat{\rho}_{2}\left\|\dot{\mathbf{q}}^{1}\right\|\left\|\dot{\mathbf{q}}_{r}^{1}\right\|+\hat{\rho}_{3}\right) \frac{\mathbf{s}_{1}}{\left\|\mathbf{s}_{1}\right\|} \\
& +\mathbf{s}_{1}^{T}\left(-A \ddot{\mathbf{q}}_{r}^{1}-L^{T} B_{1} \dot{\mathbf{q}}_{r}^{1}-L^{T} G\right)+\frac{1}{2} \mathbf{s}_{1}^{T}\left(\dot{A}-2 L^{T} B_{1}\right) \mathbf{s}_{1} \\
& +\beta_{2} \sum_{i=1}^{3}\left(\rho_{i}-\hat{\rho}_{i}\right)\left(-\dot{\hat{\rho}}_{i}\right) / \eta_{i} .
\end{aligned}
$$

Using Assumptions A2 and A3, (24) becomes

$\dot{V} \leq-\mathbf{s}_{1}^{T} L^{T} K_{d} L \mathbf{s}_{1}-\beta_{2}\left(\hat{\rho}_{1}\left\|\ddot{\mathbf{q}}_{r}^{1}\right\|+\hat{\rho}_{2}\left\|\dot{\mathbf{q}}^{1}\right\|\left\|\dot{\mathbf{q}}_{r}^{1}\right\|+\hat{\rho}_{3}\right)\left\|\mathbf{s}_{1}\right\|$

$+\rho_{1} \beta_{2}\left\|\ddot{\mathbf{q}}_{r}^{1}\right\|\left\|\mathbf{s}_{1}\right\|+\rho_{2} \beta_{2}\left\|\dot{\mathbf{q}}^{1}\right\|\left\|\dot{\mathbf{q}}_{r}^{1}\right\|\left\|\mathbf{s}_{1}\right\|+\rho_{3} \beta_{2}\left\|\mathbf{s}_{1}\right\|$

$+\beta_{2} \sum_{i=1}^{3}\left(\rho_{i}-\hat{\rho}_{i}\right)\left(-\dot{\hat{\rho}}_{i}\right) / \eta_{i}$

$\leq-\mathbf{s}_{1}^{T} L^{T} K_{d} L \mathbf{s}_{1} \leq 0$. 
From (23) and (25), it is evident that $\mathbf{s}_{1} \in L_{\infty}^{n-m} \cap$ $L_{2}^{n-m}$ and $\hat{\rho}_{i} \in L_{\infty}, i=1,2,3$. Moreover, $\mathbf{e}_{m} \rightarrow 0$ as $t \rightarrow \infty$. Also $\mathbf{q}_{d}^{2}=\sigma\left(\mathbf{q}_{d}^{1}\right)$, which implies $\mathbf{q}^{2} \rightarrow \mathbf{q}_{d}^{2}$ if $\mathbf{q}^{1} \rightarrow \mathbf{q}_{d}^{1}$, therefore, $\mathbf{q} \rightarrow \mathbf{q}_{d}$ as $t \rightarrow \infty$.

Since $\mathbf{s}_{1}$, therefore, $\mathbf{e}_{m}$ and $\dot{\mathbf{e}}_{m}$, and $\hat{\rho}_{i}$ are bounded, it follows that that $\mathbf{q}_{1}, \dot{\mathbf{q}}_{1}, \dot{\mathbf{q}}_{r}^{1}$, and $\ddot{\mathbf{q}}_{r}^{1}$ are all bounded. Therefore, all signals on the right side of (22) are bounded and we can conclude that $\dot{\mathbf{s}}_{1}$ is bounded. Using (15) allows us to conclude that $\ddot{q}_{1}$ is bounded. Substituting the control (16) into reduced-order dynamic model (11) yields

$$
\begin{aligned}
J^{T}(\lambda & \left.-\lambda_{c}\right) \\
& =\left[D\left(\mathbf{q}^{1}\right) L\left(\mathbf{q}^{1}\right) \ddot{\mathbf{q}}^{1}+B_{1}\left(\mathbf{q}^{1}, \dot{\mathbf{q}}^{1}\right) \dot{\mathbf{q}}^{1}+G\left(\mathbf{q}^{1}\right)+K_{d} L \mathbf{s}_{1}\right. \\
& \left.+\frac{\beta_{2}}{\beta_{1}} L\left(\hat{\rho}_{1}\left\|\ddot{\mathbf{q}}_{r}^{1}\right\|+\hat{\rho}_{2}\left\|\dot{\mathbf{q}}^{1}\right\|\left\|\mathbf{q}_{r}^{1}\right\|+\hat{\rho}_{3}\right) \frac{\mathbf{s}_{1}}{\left\|\mathbf{s}_{1}\right\|}\right] \\
& =\zeta_{0}\left(\mathbf{q}_{1}, \dot{\mathbf{q}}_{1}, \dot{\mathbf{q}}_{r}^{1}, \ddot{\mathbf{q}}_{r}^{1}, \hat{\rho}_{1}, \hat{\rho}_{2}, \hat{\rho}_{3}\right) .
\end{aligned}
$$

of Theorem 1. The control input will rise to whatever level is necessary to ensure the stability of the overall system.

3) $\beta_{1}$ and $\beta_{2}$ given in Assumption A2 should be chosen in such a way so as to reduce the magnitudes of $\beta_{1} / \beta_{2}$. This, in turn, reduces the magnitude of the control law.

4) No perfect force tracking objective is warranted. Nevertheless, $e_{\lambda}$ has a particular value, because improved steady-state constraint force accuracy is obtained with sufficiently high force gain. This result is similar to the results presented in [1].

5) While assuring the desired behavior, the control law (16) is discontinuous across the sliding surface $\mathbf{s}_{1}$, which leads to control chattering. Chattering is, in general, highly undesirable in practice, since it involves extremely high control activity and, further, may excite high-frequency dynamics neglected during modeling [11]. This can be remedied by smoothing out the control discontinuities in a boundary layer neighboring the sliding surface. That is

$$
\mathbf{u}=\left\{\begin{array}{lll}
-K_{d} L \mathbf{s}_{1}-\frac{\beta_{2}}{\beta_{1}} L\left(\hat{\rho}_{1}\left\|\dot{\mathbf{q}}_{r}\right\|+\hat{\rho}_{2}\left\|\dot{\mathbf{q}}^{1}\right\|\left\|\dot{\mathbf{q}}_{r}^{1}\right\|+\hat{\rho}_{3}\right) \frac{\mathbf{s}_{1}}{\left\|\mathbf{s}_{1}\right\|}-J^{T}\left(\mathbf{q}^{1}\right) \lambda_{c} & \text { if } \quad\left\|\mathbf{s}_{1}\right\| \geq \epsilon \\
-K_{d} L \mathbf{s}_{1}-\frac{\beta_{2}}{\beta_{1}} L\left(\hat{\rho}_{1}\left\|\dot{\mathbf{q}}_{r}^{1}\right\|+\hat{\rho}_{2}\left\|\dot{\mathbf{q}}^{1}\right\|\left\|\dot{\mathbf{q}}_{r}^{1}\right\|+\hat{\rho}_{3}\right) \frac{\mathbf{s}_{1}}{\epsilon}-J^{T}\left(\mathbf{q}^{1}\right) \lambda_{c} & \text { if } \quad\left\|\mathbf{s}_{1}\right\| \leq \epsilon
\end{array}\right.
$$

Therefore, $\zeta_{0}$ is a bounded function. Using the definition of $\dot{\mathbf{q}}_{r}^{1}$ and $\hat{\rho}_{i}$ again allows $\zeta_{0}$ to be rewritten as

$$
J^{T}\left(\lambda-\lambda_{c}\right)=\zeta\left(\mathbf{q}_{1}, \dot{\mathbf{q}}_{1}, \ddot{\mathbf{q}}_{1}, \mathbf{q}_{d}^{1}, \dot{\mathbf{q}}_{d}^{1}, \ddot{\mathbf{q}}_{d}^{1}\right)
$$

where $\zeta$ is a bounded function. Thus

$$
J^{T} \mathbf{e}_{\lambda}=\left(K_{\lambda}+I\right)^{-1} \zeta
$$

and the force tracking error $\left(\mathbf{f}-\mathbf{f}_{d}\right)$ are bounded and can be adjusted by changing the feedback gain $K_{\lambda}$. Thus, the theorem is proved.

\section{REMARKS}

1) Except the knowledge of constraint functions (4) and forward kinematics (5), which are invariably assumed in the literature, the proposed control laws (16) and (19)-(21) do not require the detailed description of the robot dynamics, and avoid the difficulty linked to the calculations of nonlinear dynamic functions. So the controller is very general and structurally simple as well as computationally fast.

2) For the control design, the existence of $\rho_{i}$, $i=1,2,3$, is necessary to guarantee the stability of the closed-loop system. However, these constants are not explicitly involved in the control inputs; the existence of $\rho_{i}, i=1,2,3$, is sufficient for the validity where $\epsilon>0$. By applying (29), we guarantee the attractiveness of the boundary layer. For the region inside the boundary, it can be proved that this will guarantee the ultimate boundedness of the system to within any neighborhood of the boundary layer [2].

\section{ROBUSTNESS TO INPUT DISTURBANCES}

Consider now the situation where disturbances are added to the control input. Let the robot dynamics (11) now be represented as

$$
D\left(\mathbf{q}^{1}\right) L\left(\mathbf{q}^{1}\right) \ddot{\mathbf{q}}^{1}+B_{1}\left(\mathbf{q}^{1}, \dot{\mathbf{q}}^{1}\right) \dot{\mathbf{q}}^{1}+G\left(\mathbf{q}^{1}\right)=\mathbf{u}+f+\mathbf{u}_{d}
$$

where $\mathbf{u}_{d} \in R^{n}$ is the vector of uncertainties representing torque disturbances.

Generally, in the variable structure controller design the disturbances are assumed to be bounded. But this assumption may be restrictive since such disturbances may appear as a result of unmodeled robot forces such as friction. Generally speaking, the disturbances are functions of the system states and may grow beyond any constant bounds if the system becomes unstable [12]. Therefore, we make the following assumption. 
Assumption A4. The effects of input disturbances are assumed to satisfy the following:

$$
\left\|L^{T} \mathbf{u}_{d}\right\| \leq \beta_{2} \delta_{0}+\beta_{2} \delta_{1}\left\|\mathbf{s}_{1}\right\|
$$

where $\delta_{0} \geq 0, \delta_{1} \geq 0$ are constants.

REMARK This assumption is quite reasonable as far as the effects of friction forces or some other causes are concerned since it is assumed to be unbounded and fast varying.

Concerning the disturbances, the following theorem is presented.

THEOREM 2 Consider the control law (16) with the updated laws (19)-(21) in closed-loop with the constrained manipulator modeled in (30). If $K_{d}$ in (16) is chosen such that $\gamma_{\min }\left(L^{T} K_{d} L\right)>\beta_{2} \delta_{1}$ then, the following still holds:

1) $\mathbf{q} \rightarrow \mathbf{q}_{d}$ as $t \rightarrow \infty$.

2) Steady-state force $\left(\mathbf{f}-\mathbf{f}_{d}\right)$ is bounded and inversely proportional to the norm of the matrix $\left(K_{\lambda}+I\right)$.

PROOF. Similar to the derivation in the proof of Theorem 1, we have

$$
\begin{aligned}
A \dot{\mathbf{s}}_{1}= & L^{T} D L \dot{\mathbf{s}}_{1}=L^{T} \mathbf{u}-A \ddot{\mathbf{q}}_{r}^{1}-L^{T} B_{1} \dot{\mathbf{q}}_{r}^{1} \\
& -L^{T} G-L^{T} B_{1} \mathbf{s}_{1}+L^{T} \mathbf{u}_{d}
\end{aligned}
$$

Reconsidering the nonnegative function

$$
V(t)=\frac{1}{2} \mathbf{s}_{1}^{T} A \mathbf{s}_{1}+\frac{1}{2}\left(\sum_{i=1}^{3}\left(\bar{\rho}_{i}-\hat{\rho}_{i}\right)^{2} / \eta_{i}\right) \beta_{2}
$$

where $\bar{\rho}_{1}=\rho_{1}>0, \bar{\rho}_{2}=\rho_{2}>0, \bar{\rho}_{3}=\rho_{3}+\delta_{0}>0, \rho_{i}$, $i=1,2,3$, are defined in (14).

Differentiating (33) with respect to time along the solution of (32) and using Properties 3 and 4 give

$$
\begin{aligned}
\dot{V}= & \mathbf{s}_{1}^{T} A \dot{\mathbf{s}}_{1}+\frac{1}{2} \mathbf{s}_{1}^{T} \dot{A} \mathbf{s}_{1}+\beta_{2} \sum_{i=1}^{3}\left(\bar{\rho}_{i}-\hat{\rho}_{i}\right)\left(-\dot{\hat{\rho}}_{i}\right) / \eta_{i} \\
= & \mathbf{s}_{1}^{T}\left(L^{T} \mathbf{u}-A \ddot{\mathbf{q}}_{r}^{1}-L^{T} B_{1} \dot{\mathbf{q}}_{r}^{1}-L^{T} G-L^{T} B_{1} \mathbf{s}_{1}+L^{T} \mathbf{u}_{d}\right) \\
& +\frac{1}{2} \mathbf{s}_{1}^{T}\left(\frac{d}{d t} A\right) \mathbf{s}_{1}+\beta_{2} \sum_{i=1}^{3}\left(\bar{\rho}_{i}-\hat{\rho}_{i}\right)\left(-\dot{\rho}_{i}\right) / \eta_{i} \\
= & -\mathbf{s}_{1}^{T} L^{T} K_{d} L \mathbf{s}_{1}-\frac{\beta_{2}}{\beta_{1}} \mathbf{s}_{1}^{T} L^{T} L\left(\hat{\rho}_{1}\left\|\ddot{\mathbf{q}}_{r}^{1}\right\|+\hat{\rho}_{2}\left\|\dot{\mathbf{q}}^{1}\right\|\left\|\dot{\mathbf{q}}_{r}^{1}\right\|+\hat{\rho}_{3}\right) \frac{\mathbf{s}_{1}}{\left\|\mathbf{s}_{1}\right\|} \\
& +\mathbf{s}_{1}^{T}\left(-A \ddot{\mathbf{q}}_{r}^{1}-L^{T} B_{1} \dot{\mathbf{q}}_{r}^{1}-L^{T} G+L^{T} \mathbf{u}_{d}\right) \\
& +\frac{1}{2} \mathbf{s}_{1}^{T}\left(\dot{A}-2 L^{T} B_{1}\right) \mathbf{s}_{1} \\
& +\beta_{2} \sum_{i=1}^{3}\left(\bar{\rho}_{i}-\hat{\rho}_{i}\right)\left(-\dot{\rho}_{i}\right) / \eta_{i}
\end{aligned}
$$

Using Assumptions A2, A3, and A4, (34) becomes

$$
\begin{aligned}
\dot{V} \leq & -\mathbf{s}_{1}^{T} L^{T} K_{d} L \mathbf{s}_{1}-\beta_{2}\left(\hat{\rho}_{1}\left\|\ddot{\mathbf{q}}_{r}^{1}\right\|+\hat{\rho}_{2}\left\|\dot{\mathbf{q}}^{1}\right\|\left\|\dot{\mathbf{q}}_{r}^{1}\right\|+\hat{\rho}_{3}\right)\left\|\mathbf{s}_{1}\right\| \\
& +\rho_{1} \beta_{2}\left\|\ddot{\mathbf{q}}_{r}^{1}\right\|\left\|\mathbf{s}_{1}\right\|+\rho_{2} \beta_{2}\left\|\dot{\mathbf{q}}^{1}\right\|\left\|\dot{\mathbf{q}}_{r}^{1}\right\|\left\|\mathbf{s}_{1}\right\| \\
& +\rho_{3} \beta_{2}\left\|\mathbf{s}_{1}\right\|+\beta_{2} \delta_{0}\left\|\mathbf{s}_{1}\right\|+\beta_{2} \delta_{1}\left\|\mathbf{s}_{1}\right\|^{2} \\
& +\beta_{2} \sum_{i=1}^{3}\left(\bar{\rho}_{i}-\hat{\rho}_{i}\right)\left(-\dot{\hat{\rho}}_{i}\right) / \eta_{i} \\
\leq & -\left(\gamma_{\min }\left(L^{T} K_{d} L\right)-\beta_{2} \delta_{1}\right)\left\|\mathbf{s}_{1}\right\|^{2} \leq 0 .
\end{aligned}
$$

then using the same argument as that in the Proof of Theorem 1, we have the conclusion.

\section{SIMULATION RESULTS}

A two-link robotic manipulator with a circular path constraint, as given in $[19,13,14]$, is used to verify the validity of the control approach outlined here. The original model, in the form of (1), can be written as

$$
\begin{aligned}
& {\left[\begin{array}{ll}
D_{11}\left(q_{2}\right) & D_{12}\left(q_{2}\right) \\
D_{12}\left(q_{2}\right) & D_{22}\left(q_{2}\right)
\end{array}\right]\left[\begin{array}{l}
\ddot{q}_{1} \\
\ddot{q}_{2}
\end{array}\right]} \\
& +\left[\begin{array}{cc}
-F_{12}\left(q_{2}\right) \dot{q}_{2} & -F_{12}\left(q_{2}\right)\left(\dot{q}_{1}+\dot{q}_{2}\right) \\
F_{12}\left(q_{2}\right) \dot{q}_{1} & 0
\end{array}\right]\left[\begin{array}{l}
\dot{q}_{1} \\
\dot{q}_{2}
\end{array}\right] \\
& +\left[\begin{array}{l}
g_{1}\left(q_{1}, q_{2}\right) g \\
g_{2}\left(q_{1}, q_{2}\right) g
\end{array}\right]=\left[\begin{array}{l}
u_{1} \\
u_{2}
\end{array}\right]+f
\end{aligned}
$$

where

$$
\begin{aligned}
& D_{11}\left(q_{2}\right)=\left(m_{1}+m_{2}\right) l_{1}^{2}+m_{2} l_{2}^{2}+2 m_{2} l_{1} r_{2} \cos \left(q_{2}\right) \\
& D_{12}\left(q_{2}\right)=m_{2} l_{2}^{2}+m_{2} l_{1} l_{2} \cos \left(q_{2}\right) \\
& D_{22}\left(q_{2}\right)=m_{2} l_{2}^{2} \\
& F_{12}\left(q_{2}\right)=m_{2} l_{1} l_{2} \sin \left(q_{2}\right) \\
& g_{1}\left(q_{1}, q_{2}\right)=-\left(m_{1}+m_{2}\right) l_{1} \cos \left(q_{1}\right)-m_{2} l_{2} \cos \left(q_{1}+q_{2}\right) \\
& g_{2}\left(q_{1}, q_{2}\right)=-m_{2} l_{2} \cos \left(q_{1}+q_{2}\right) .
\end{aligned}
$$

The parameter values used are the same as those [14]

$$
\begin{aligned}
l_{1} & =1, & l_{2} & =0.8 ; \\
m_{1} & =0.5 \mathrm{~kg}, & m_{2} & =0.5 \mathrm{~kg} ; \\
J_{1} & =5 \mathrm{~kg} . \mathrm{m}, & J_{2} & =5 \mathrm{Kg} . \mathrm{m} .
\end{aligned}
$$

The constraint is a circle in the work space (the $x-y$ plane) whose center coincides with the axis of rotation of the first link. Fig. 1 depicts the two-link manipulator and the constraint. The constraint surface is expressed mathematically as

$$
\phi(p)=x^{2}+y^{2}-r^{2}=0, \quad p=\left[\begin{array}{ll}
x & y
\end{array}\right]^{T} .
$$




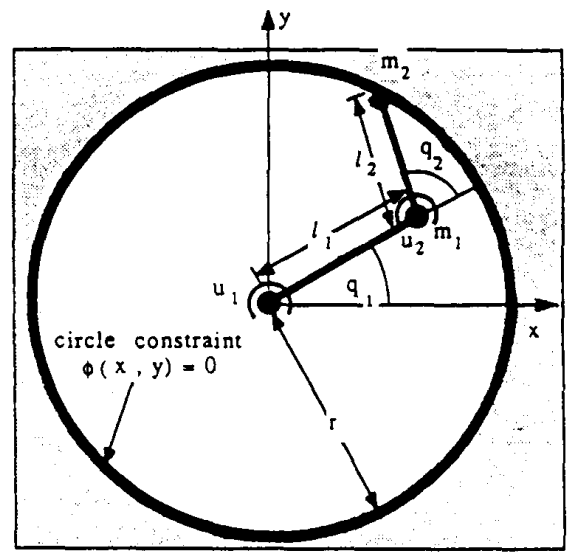

Fig. 1. Two-link manipulator and circle constraint.

The transformation from work space to joint space is given by

$$
H(q)=\left[\begin{array}{l}
l_{1} \cos \left(q_{1}\right)+l_{2} \cos \left(q_{1}+q_{2}\right) \\
l_{1} \sin \left(q_{1}\right)+l_{2} \sin \left(q_{1}+q_{2}\right)
\end{array}\right] .
$$

The constraint, when expressed in terms of joint space,

$$
\psi(q)=l_{1}^{2}+l_{2}^{2}+2 l_{1} l_{2} \cos q_{2}-r^{2}=0
$$

which has an unique constant solution for $q_{2}$

$$
q_{2}=\cos ^{-1}\left(\frac{r^{2}-\left(l_{1}^{2}+l_{2}^{2}\right)}{2 l_{1} l_{2}}\right)=q_{2}^{*} .
$$

The Jacobian matrix of (39) is

$$
J(q)=\left[\begin{array}{c}
0 \\
-2 l_{1} l_{2} \sin \left(q_{2}\right)
\end{array}\right] .
$$

Therefore the matrix $L$ defined in (10) is

$$
L\left(q^{1}\right)=\left[\begin{array}{ll}
1 & 0
\end{array}\right]^{T} .
$$

The constrained robot motion equation (11), when restricted to the circle, can be expressed as

$$
\begin{gathered}
{\left[\begin{array}{l}
D_{11}\left(q_{2}^{*}\right) \\
D_{22}\left(q_{2}^{*}\right)
\end{array}\right] \dot{q}_{1}+\left[\begin{array}{c}
0 \\
F_{12}\left(q_{2}^{*}\right) \dot{q}_{1}
\end{array}\right]+\left[\begin{array}{l}
g_{1}\left(q_{1}, q_{2}^{*}\right) g \\
g_{2}\left(q_{1}, q_{2}^{*}\right) g
\end{array}\right]} \\
=\left[\begin{array}{l}
u_{1} \\
u_{2}
\end{array}\right]+\left[\begin{array}{c}
0 \\
-2 l_{1} l_{2} \sin \left(q_{2}^{*}\right)
\end{array}\right] \lambda .
\end{gathered}
$$

The constraint forces are

$$
\begin{aligned}
& f_{1}=0, \\
& f_{2}=-2 l_{1} l_{2} \sin \left(q_{2}^{*}\right) \lambda,
\end{aligned}
$$

The control objective is to determine a feedback control so that the joint $q_{1}$ tracks the desired trajectory $q_{1 d}$ and maintains the constraint force $f_{2}$ to the desired $f_{d}$, where $q_{1 d}$ and $f_{d}$ are assumed to be consistent with the imposed constraint.

Since $\lambda \rightarrow \lambda_{d}$ means $f_{2} \rightarrow f_{d}$, hence in this simulation, $q_{1 d}$ and $f_{d}$ are chosen as

$$
\begin{aligned}
& q_{1 d}=-90+52.5(1-\cos (1.26 t)) \\
& \lambda_{d}=10 .
\end{aligned}
$$

The two control parameters $\beta_{1}$ and $\beta_{2}$ are chosen as $\beta_{1}=\beta_{2}=1$. The position control gain is chosen as $K_{d}=5$, and the force control gain $K_{\lambda}=0.8$. The adaptive gain are chosen as $\eta_{1}=4.5, \eta_{2}=4.5, \eta_{2}=4.5$.

Since trajectory tracking on the constrained surface with specified constraint force is of interest, the initial position and velocity of manipulator are chosen on the desired trajectory.

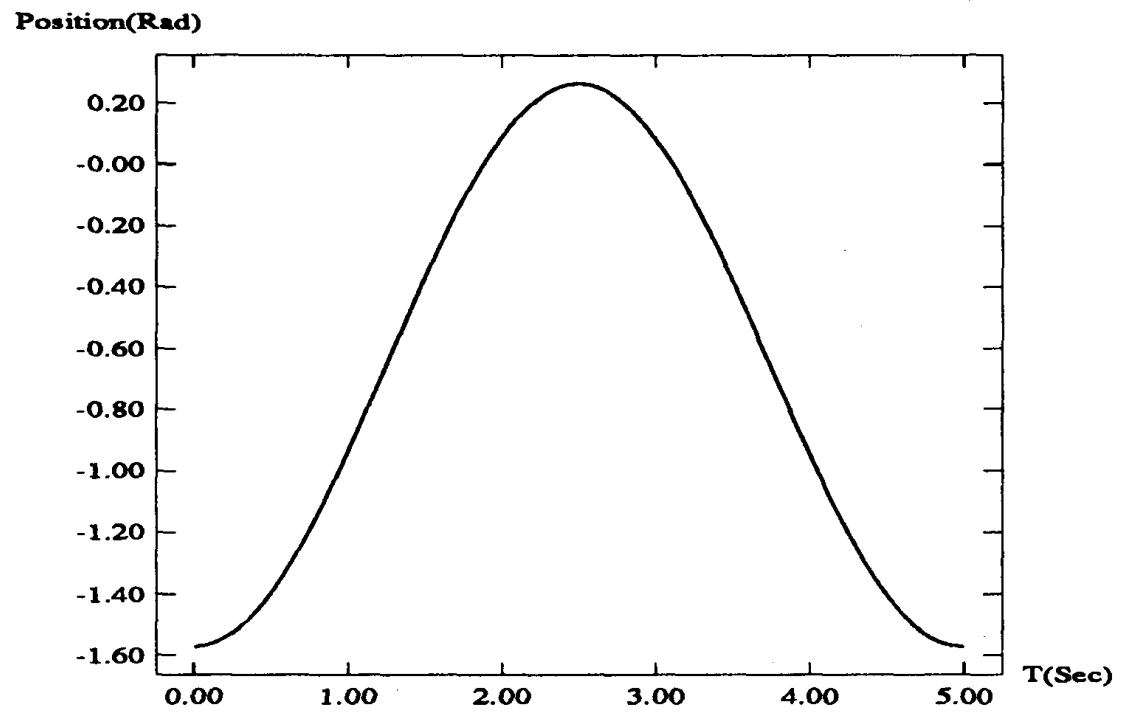

Fig. 2. Desired trajectory. 

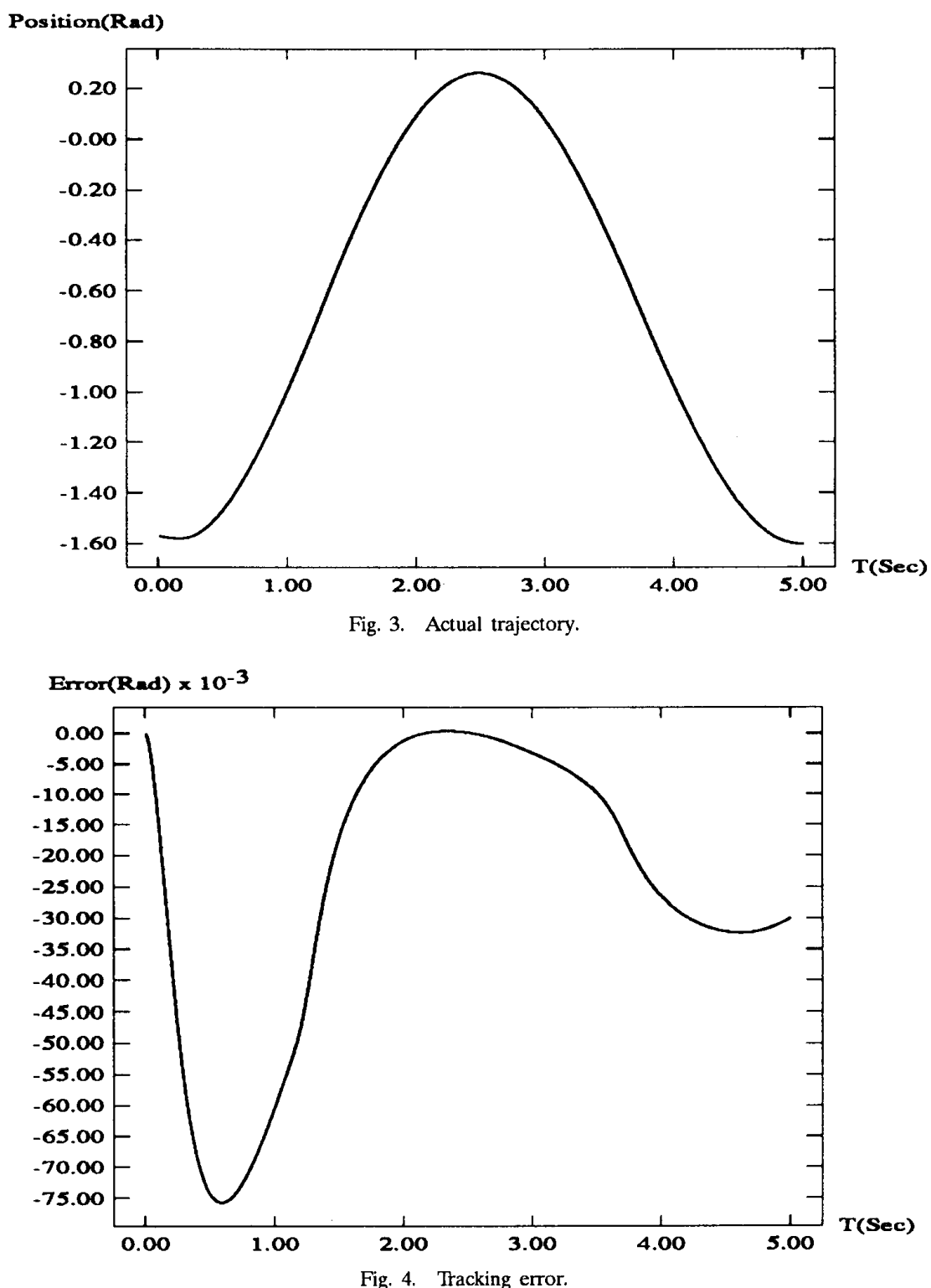

$$
\begin{array}{ll}
q_{1}(0)=-90 ; & q_{2}(0)=80 ; \\
\dot{q}_{1}(0)=0 ; & \dot{q}_{2}(0)=0 .
\end{array}
$$

The initial constraint force is assumed as $f_{2}=0$, i.e., $\lambda=0$. The initial control gain $\hat{\rho}_{i}(0), i=1,2,3$, are taken as $\hat{\rho}_{1}(0)=2, \hat{\rho}_{2}(0)=1, \hat{\rho}_{3}(0)=1$. In order to reduce the control chattering, the boundary layer is chosen as $\varepsilon=0.05$.

The results of the simulation are shown in Figs. 2-8. Fig. 2 shows the desired joint trajectory, Fig. 3 shows the actual trajectory of joint 1 , and Fig. 4 shows its tracking error. Fig. 5 shows contact force $\lambda$. The final maximum error with $\lambda_{d}$ is $1.5 N$. Figs. 6 and 7 show the torques exerted at manipulator joints, and sliding surface is shown in Fig. 8. These results show that the control objective is achieved successfully.

\section{CONCIUSIONS}

Based on the reduced formulation of constrained robots given in $[13,14]$, an efficient adaptive variable structure tracking control algorithm, to achieve trajectory tracking of an end-effector on a constrained holonomic smooth surface with specified constraint forces, is presented in this paper. In the method, no exact knowledge about robot dynamics is needed, 

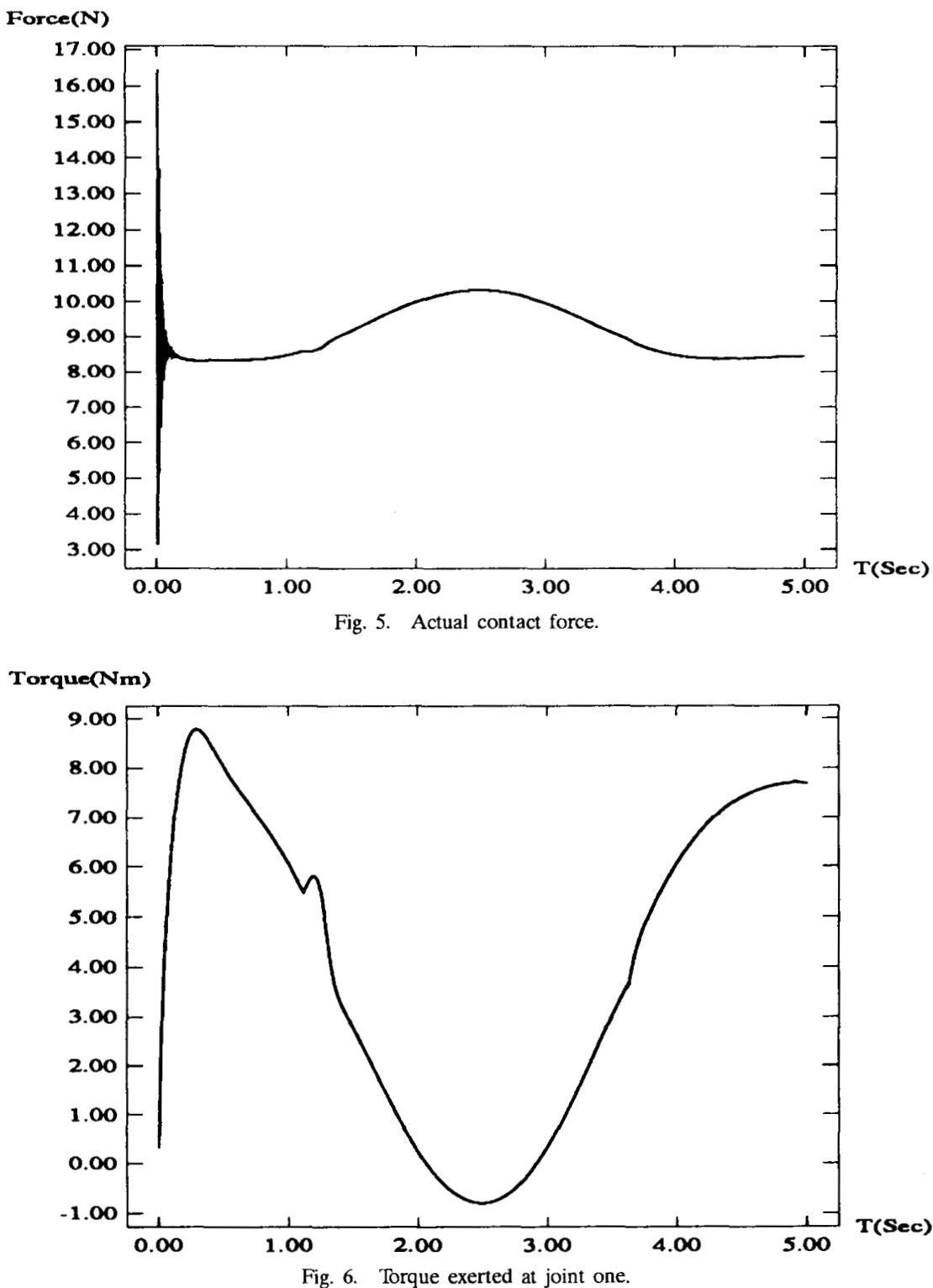

and the calculation of nonlinear dynamic functions (regressor matrix) is avoided. Furthermore, the number of the control parameters needed for nonlinear compensation is three at most, and that number is determined independently of the number of degrees of freedom of the robotic manipulator. The robustness analysis has shown that in the presence of a class of dynamic (state-dependent) uncertainties, the control law still provides stable tracking. A simple two-link manipulator and a circle constraint has been used to illustrate the methodology developed in this paper, and the simulation results are quite satisfactory.

In developing the control strategy, it is assumed that the description of constraint functions are exact. However, in some situations, the constraint functions may not be known exactly. Hence, extension of the results to the case in which constraint functions are not totally known is an interesting further research topic.

\section{REFERENCES}

[1] Carelli, R., and Kelly, R. (1991)

An adaptive impedance/force controller for robot manipulators.

IEEE Transactions on Automatic Control, 36 (1991), 967-971.

[2] Corless, M. J., and Leitmann, G. (1981)

Continuous state feedback guaranteeing uniform ultimate boundedness for uncertain dynamic system.

IEEE Transactions on Automatic Control, 26 (1981), 1139-1144. 


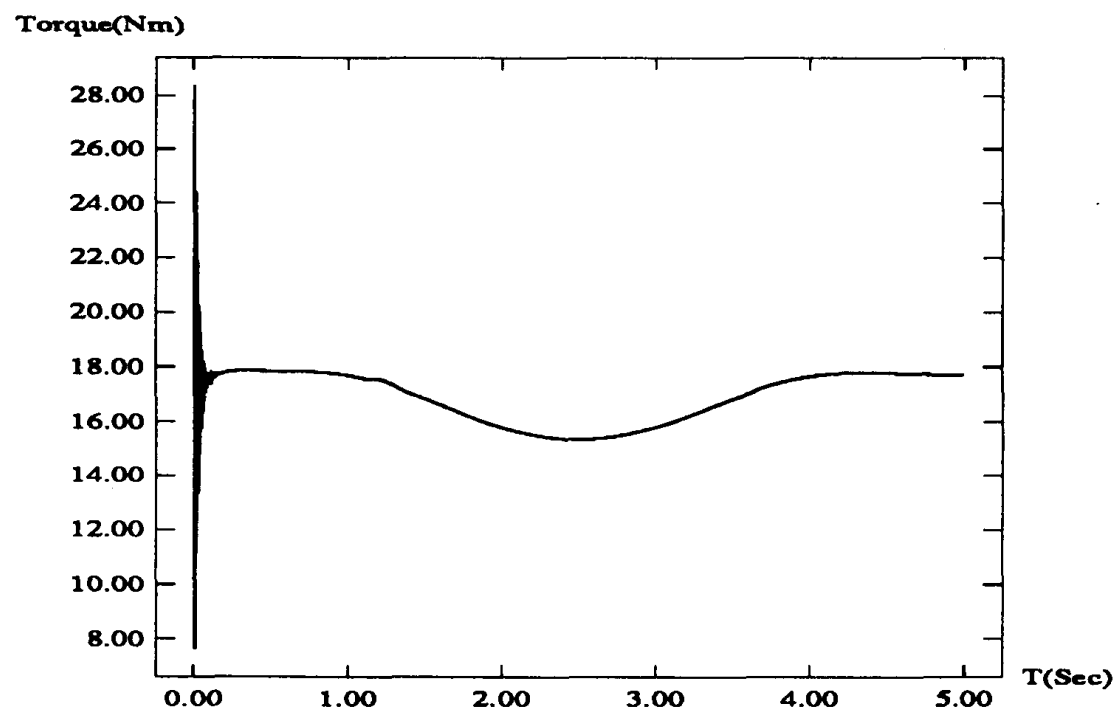

Fig. 7. Torque exerted at joint two.

$\times 10^{-3}$

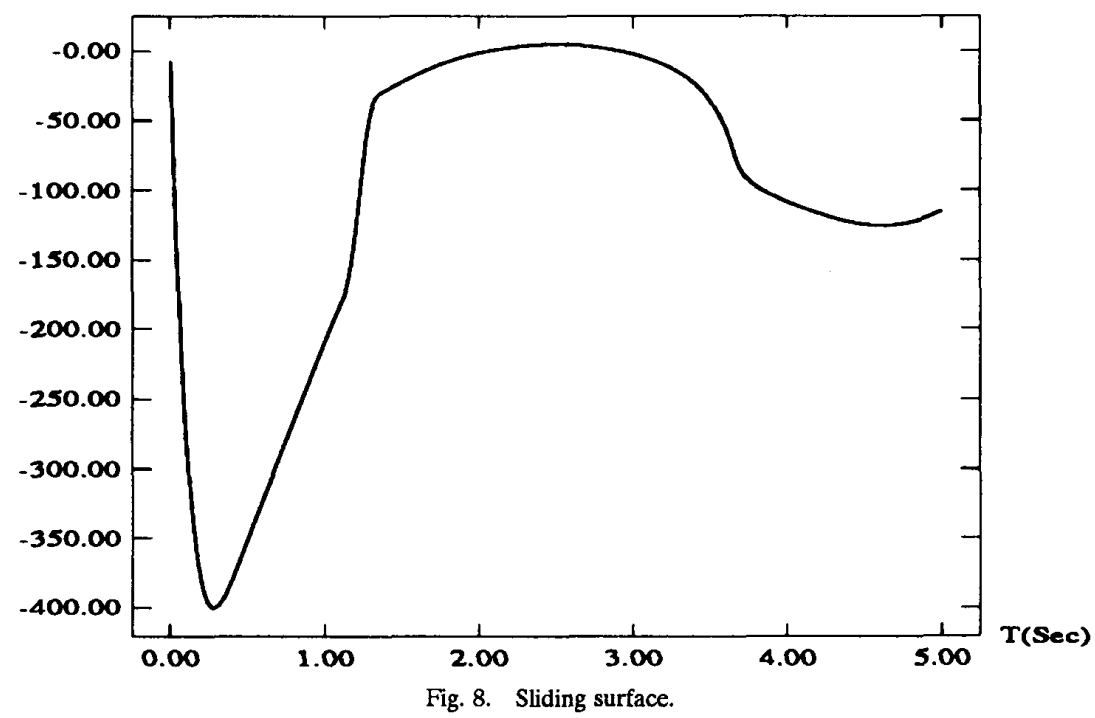

[3] Hemami, H., and Wyman, B. F. (1979)

Modeling and control of constrained dynamic system with application to biped locomotion in the frontal plane. IEEE Transactions on Automatic Control, AC-24 (1979), 526-535.

[4] Huang, H. P., and Lin, M. (1990)

Variable structure control of constrained dynamic systems. In Proceedings of IEEE International Conference on Robotics and Automation (1990), 1362-1367.

[5] Leung, T. P., Zhou, Q. J., and Su, C. Y. (1991)

An adaptive variable structure model following control design for robot manipulators.

IEEE Transactions on Automatic Control, 36 (1991), 347-353.

[6] Lui, L., Han, Y., Lingarkar, R., Sinha, N. A., and Elbestawi, M. A. (1989)

On adaptive force/motion control of constrained robots. In Proceedings of IECON'89 (1989), 433-438.
[7] McClamroch, N. H., and Huang, H. P. (1985) Dynamics of a closed chain manipulator. In Proceedings of American Control Conference (1985), 50-55.

[8] McClamroch, N. H., and Wang, D. (1988) Feedback stabilization and tracking of constrained robots. IEEE Transactions on Automatic Control, 33 (1988), 419-426.

[9] Mills, J. K., and Goldenberg, A. A. (1989) Force and position control of manipulators during constrained motion tasks. IEEE Transactions on Robotics and Automation, 5 (1989), $30-46$.

[10] Slotine, J. J. E., and Li, W. (1987) On the adaptive control of robot manipulator. International Journal of Robotics Research, 6, 3 (1987), 49-59. 
[11] Slotine, J. J. E., and Sastry, S. S. (1983)

Tracking control of nonlinear system using sliding surface, with application to robot manipulators. International Journal of Control, 38 (1983), 465-492.

[12] Stepanenko, Y., and Yuan, J. (1992) Robust adaptive control of a class of nonlinear mechanical systems with unbounded and fast-varying uncertainties. Automatica, 24 (1992), 265-276.

[13] Su, C. Y., Leung, T. P., and Zhou, Q. J. (1990) Adaptive control of robot manipulators under constrained motion.

In Proceedings of the 29th IEEE Conference on Decision and Control (1990), 2650-2655.

[14] Su, C. Y., Leung, T. P., and Zhou, Q. J. (1992) Force/motion control of constrained robots using sliding mode.

IEEE Transactions on Automatic Control, 37 (1992), 668-672.

[15] Tomei, P. (1991)

Adaptive PD controller for robot manipulators.

IEEE Transactions on Robotics and Automation, 7 (1991), 565-570.

[16] Utkin, V. I. (1983)

Variable structure systems-Present and future.

Automation and Remote Control, 44 (1983), 1105-1120.
[17] Xu, J. X., Boesch, M. A., and Young, K. D. (1991)

Variable structure control of rigid-flexible closed-chain systems.

In Proceedings of American Control Conference (1991), 1903-1908.

[18] Yoon, C. S., and Salam, F. M. A. (1989)

Compliant control of constrained robot manipulators: Stabilization on the constraint surface.

In Proceedings of the 28th IEEE Conference on Decision and Control (1989), 1622-1627.

[19] Young, K. D. (1988)

Applications of sliding mode to constrained robot motion control.

In Proceedings of American Control Conference (1988), 912-917.

[20] Yun, X. (1988)

Dynamic state feedback control of constrained robot manipulator.

In Proceedings of the 27th IEEE Conference on Decision and Control (1988), 433-438.
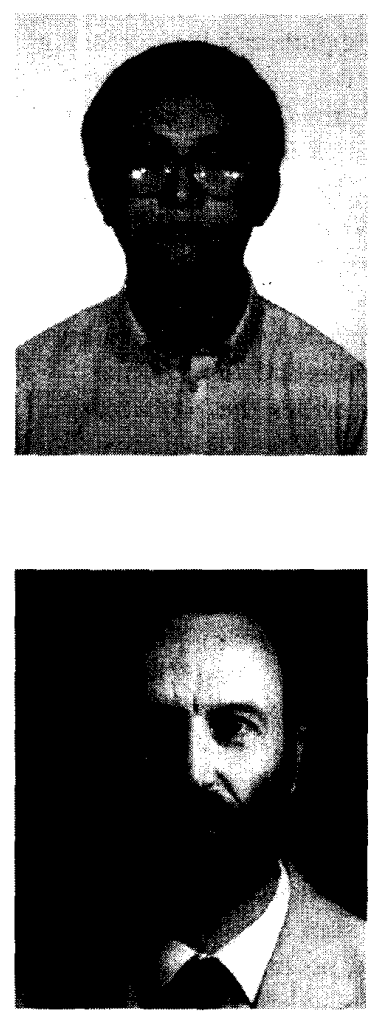

Chun-Yi Su (M'93) was born in Beijing, People's Republic of China, in 1962 He received the B.E. degree in control engineering from Shaanxi Institute of Mechanical Engineering, People's Republic of China, in 1982 and the M.E. and $\mathrm{Ph} . \mathrm{D}$. degrees in control engineering from the South China University of Technology, People's Republic of China, in 1987 and 1990, respectively.

$\mathrm{He}$ is currently a Research Associate with the Department of Mechanical Engineering, University of Victoria, Canada. His research interests are in the area of control of nonlinear feedback systems, and include applications to control of mechanical systems.

Yury Stepanenko received his Ph.D. degree in 1964 from the Moscow Institute of Machine Science, Moscow, Russia. He was awarded a second degree, Doctor of Science, in 1972, also from the Moscow Institute of Machine Science.

His professional record includes the positions of Senior Researcher in the Moscow Institute of Machine Science, Professor and Chairman of the Department of Automatic Control at the Institute of Machine-Tool Engineering, Moscow, and Senior Consultant in ISKRA Corporation, Lyublyana, Sloveniya. Currently, he is a Professor of Mechanical Engineering University of Victoria, Victoria, B.C. $\mathrm{He}$ is also a project leader at the Institute for Robotics and Intelligent Systems, Canada. His research interests are in dynamics and control of nonlinear systems and robotics.

Dr. Stepanenko is the author of more than 70 scientific papers and a monograph entitled "Dynamics of Spatial Mechanisms" and holds ten patents. 